\title{
CMS Track Reconstruction Performance
}

\section{Boris Mangano*}

University of California, San Diego

E-mail: boris.mangano@cern.ch

The CMS experiment relies on an all-silicon Tracker for the reconstruction of charged particles in the hostile radiation environment of proton collisions at the LHC at CERN. Even if the core of the tracking software has not changed in years, several important improvements have been developed recently to increase the reconstruction efficiency of charged hadrons, reduce the rate of fake tracks and refine the overall quality of the reconstructed trajectories. Despite the higher level of complexity, the tracking software is much faster than before. An outline of the recent changes to the tracking software is given and current performance is described according to the most recent studies based on Monte Carlo simulations.

17th International Workshop on Vertex detectors

July 28 - 1 August 2008

Utö Island, Sweden

* on behalf of the CMS collaboration. 


\section{Introduction}

The CMS [1] is one of the two general purpose experiments at the LHC of CERN. The LHC environment constitutes an experimental challenge: p-p collisions at the unprecedented luminosity of $10^{34} \mathrm{~cm}^{-2} \mathrm{~s}^{-1}$ will produce in the order of 20 superimposed events at a rate of $40 \mathrm{MHz}$, resulting in thousands of tracks in the acceptance of the tracking system per bunch crossing [2]. In order to ensure full functionality after exposure to 10 years of LHC operation, the CMS tracking system relies on radiation-hard silicon sensors and front-end electronics [3].

To produce a measurable curvature for high momentum particles, the tracking system is embedded in a solenoidal magnetic field of $4 \mathrm{~T}$ and the high granularity of the sensors results in a low occupancy even for the extremely high flux of charged particles expected from proton collisions at the LHC. The good spatial resolution of the innermost silicon pixel sensors translates into precise extrapolation of charged particle trajectories to the interaction region. Similarly a good estimate of the track parameters at the outer boundary of the tracker provides the link to the other detector systems, in particular the electromagnetic calorimeter and the muon chambers. Despite the care that has been taken throughout the design of the Tracker, the final material budget of the CMS silicon tracking system exceeds the originally expected value and sets additional challenges to the reconstruction software. While a description of the effects of multiple scattering and energy loss has been implemented since the initial version of the tracking software [5], recent efforts have been invested to increase the efficiency in reconstructing those many charged hadrons that suffer an inelastic nuclear interaction after they cross few layers of the tracking system. These particles produce few measurements in the Tracker and as a result they can be reconstructed only as short helix segments. In order to discriminate such genuine short trajectories from the background of accidentally aligned measurements, the track reconstruction sequence is run several times according to an iterative procedure. During the first iteration, the longer and higher momentum trajectories are detected; subsequently, additional iterations tackle the more difficult reconstruction of short and low momentum trajectories. The pattern recognition in later tracking steps is facilitated by locking all the measurements that are unambiguously associated to the trajectories already reconstructed in the previous steps.

The reconstruction of low transverse momentum trajectories is important to analyze the $p_{t}$ spectrum of the charged particles produced in LHC collisions. Such spectrum is expected to peak around $500 \mathrm{MeV} / \mathrm{c}$ and its analysis will help to understand the efficacy of different Monte Carlo generators in describing parton interactions at the unprecedented scale of energy of the LHC. Also, the tracking of low momentum trajectories and of short tracks improves the identification of $K^{0}$ and $\Lambda$ decays and the reconstruction of secondary vertices due to inelastic nuclear interactions: both aspects can improve the performance of b-tagging and particle-flow algorithms. In the following sections, the benefits of the iterative tracking approach are discussed in terms of track reconstruction efficiency, rate of fake tracks and computation speed.

The common core software used by each tracking iteration for both trajectory building and trajectory fitting has been also improved to take better into account the non-uniformity of the magnetic field in some regions of the tracker volume and to use a more accurate description of the detector material. 


\section{Detector Layout}

The CMS Silicon Tracker consists of an inner pixel system enclosed in layers of micro-strip sensors. Both subsystems comprise a central cylindrical barrel part and two end-caps. In the beam (z) direction the systems extends to $\pm 2.7 \mathrm{~m}$ from the interaction point; the outer radius is about $1.1 \mathrm{~m} \mathrm{[3].}$

The pixel system consists of three barrel layers at radii of 4.4, 7.3 and $10.2 \mathrm{~cm}$ and two sets of turbine-like endcap layers at distances of 34.5 and $46.5 \mathrm{~cm}$ on each side of the interaction region. The pixels have a size of $100 \mu \mathrm{m} \times 150 \mu \mathrm{m}$.

The rectangular strip sensors in the barrel are arranged in 10 cylindrical layers starting at a radius of about $24 \mathrm{~cm}$, the strips being parallel to the beam axis (mono configuration). The four inner layers consist of thin sensors $(320 \mu \mathrm{m})$, the six outer layers of thick sensors $(500 \mu \mathrm{m})$. The two inner layers of each of the two strip sub-structures are equipped with pairs of sensors mounted back-to-back with an angle of $100 \mathrm{mrad}$ (stereo configuration). The pitch varies between 80 and $183 \mu \mathrm{m}$ : unlike the sensors placed on intermediary barrel layers, the inter-strip distance is smaller for sensors on inner $(80 \mu \mathrm{m})$ and outer $(122 \mu \mathrm{m})$ layers. A better position resolution is indeed necessary to properly extrapolate the track parameters from the Strip Tracker towards the other sub-detectors of CMS: inward to pixel detector and outward to electromagnetic calorimeter and muon system.

In each end-cap, trapezoidal strip sensors are arranged in disks perpendicular to the beam line with the strips running radially (mono configuration). A first set of three disks complements the inner part of the barrel. A further set of nine disks extends to the outer radius of the barrel system. On the disks, sensors are arranged in up to seven rings with a radial variation of thickness and pitch similar to the one in the barrel. Rings 1,2 and 5 are equipped with modules in a stereo configuration. Given a cylindrical reference frame with the $z$-axis along the beam line of LHC, all the strip sensors provide a precise measurement of the $\phi$ coordinate. In addition, the sensors in stereo configuration provide also a less accurate estimate of either the $z$ coordinate (in the barrel) or the $\rho$ coordinate (in the end-caps). Conversely the pixel sensors provide always genuine 2dimensional measurements (Fig. 1).

Despite the care that has been taken throughout the design of the Tracker to minimize the amount of material used, the Tracking System of CMS is ultimately pretty heavy. A charged particle going through the barrel of the Tracker sees around 0.4-0.6 radiation lengths $\left(X_{0}\right)$ and 0.10.2 interaction lengths $\left(\lambda_{0}\right)$; the material budget increases up to $1.8 X_{0}$ and $0.55 \lambda_{0}$ for particles with $|\eta| \sim 1.1-1.8$ (Fig. 2). Indeed most of the services, copper wires and cables used to cool down, power and read-out the Tracker's sensors are conducted outside the tracking system through the two gaps between the barrel and two end-caps . A particle with $|\eta| \sim 1.1-1.8$ can cross the high $X_{0}$ and $\lambda_{0}$ materials placed in the barrel-encap transition region up to three times (Fig. 1).

While moderate energy $\left(p_{t}<100 \mathrm{GeV} / \mathrm{c}\right)$ muons that pass through the whole Tracker volume are affected only by Multiple Scattering and the energy losses of a Minimum Ionizing Particle (MIP), electrons can lose most of their energy by means of hard Bremsstrahlung radiation [5]. Similarly charged hadrons suffer elastic and inelastic nuclear interactions with the Tracker material: up to $10 \%$ of charged pions produced in LHC collisions will experience destructive inelastic interactions before they cross the minimum number of sensitive layers that is necessary to measure 
the curvature of their trajectories (Fig. 3).

\section{Track Reconstruction}

The core of the CMS software for reconstruction of charged particles is modularized in four

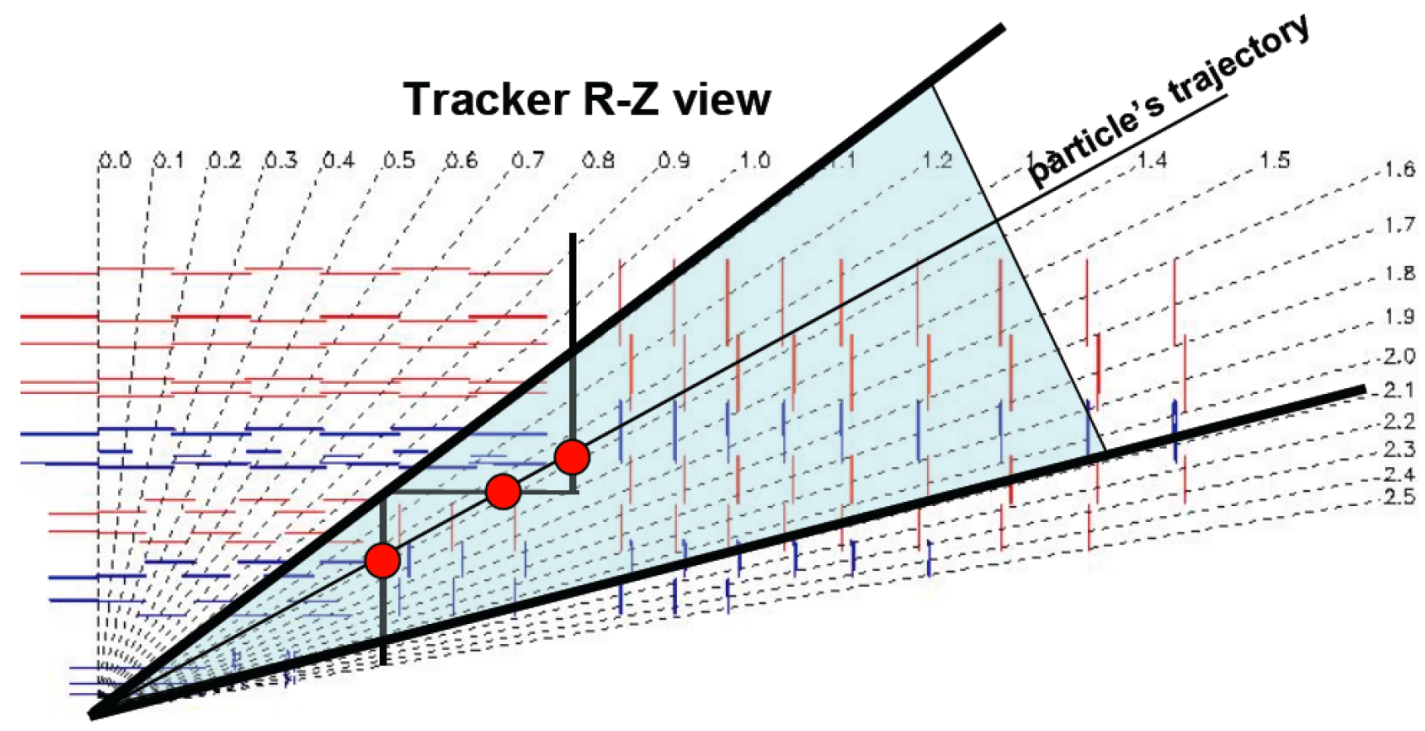

Figure 1: R-Z view of the upper-right quadrant of the CMS silicon Tracker. In red are the mono strip sensors that measure only the $\phi$ coordinate; in blue are the stereo sensors that measure both the $\phi$ and $z$ coordinates in the barrel (or the $\phi$ and $\rho$ coordinates in the end-cap). The three red spots show how a particle with 1.1 $<\eta<1.8$ can cross up to three times the services and cables that run along the gap between the barrel and end-cap structures of the Tracker.

stages [5]. The first stage provides seeds for further reconstruction, based on pairs or triplets of hits which are selected to be compatible with the interaction region and a lower $p_{t}$ limit. Due to the low occupancy and the unambiguous 2-dimensional position information, the pixel layers provide generally the best seeding. In the region of the Tracker at high $|\eta|$, pixel and strip measurements are combined to extend the geometrical acceptance of the Pixel sub-detector and provide an efficient seeding up to $|\eta| \sim 2.5$.

The second reconstruction stage uses a first estimate of the track parameters, calculated from the seed, to collect the full set of measurements associated to the same charged particle. It is based on a combinatorial Kalman filter [4] approach: starting from the current parameters, the trajectory is extrapolated to the next layer of the Tracker and compatible hits are selected based on the $\chi^{2}$ between the predicted and the measured positions. The Kalman update of the predicted parameters with each of the compatible hits provides a new set of trajectory candidates. Many candidates are built in parallel until the hits on the last layer of the Tracker are added. Ultimately, ambiguities are resolved between tracks sharing a substantial number of points. 

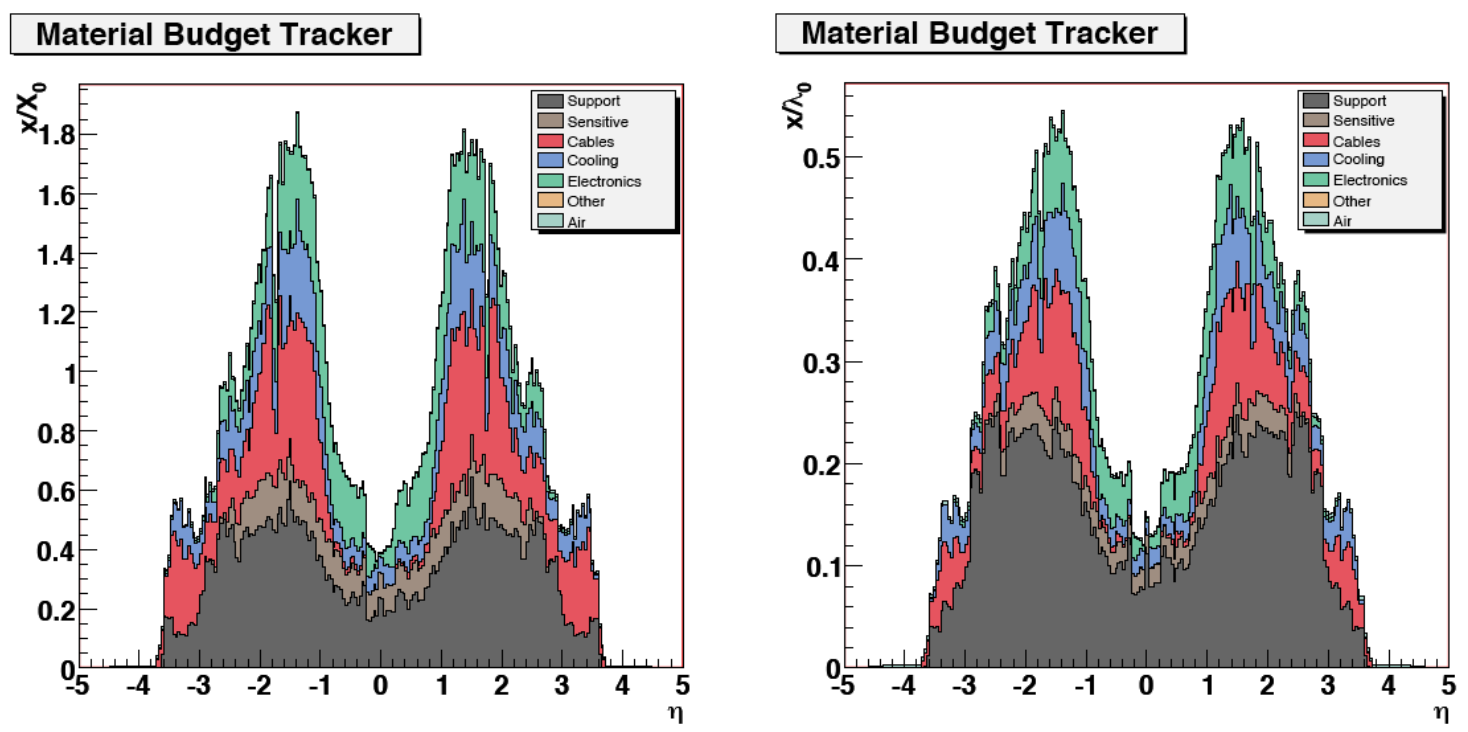

Figure 2: Distribution of the Tracker material budget versus $\eta$ expressed in terms of radiation lengths (left) and interaction lengths (right). The peak around $|\eta| \sim 1.1-1.8$ is due to all the cables and services that run along the gap between the barrel and end-cap structures of the Tracker.
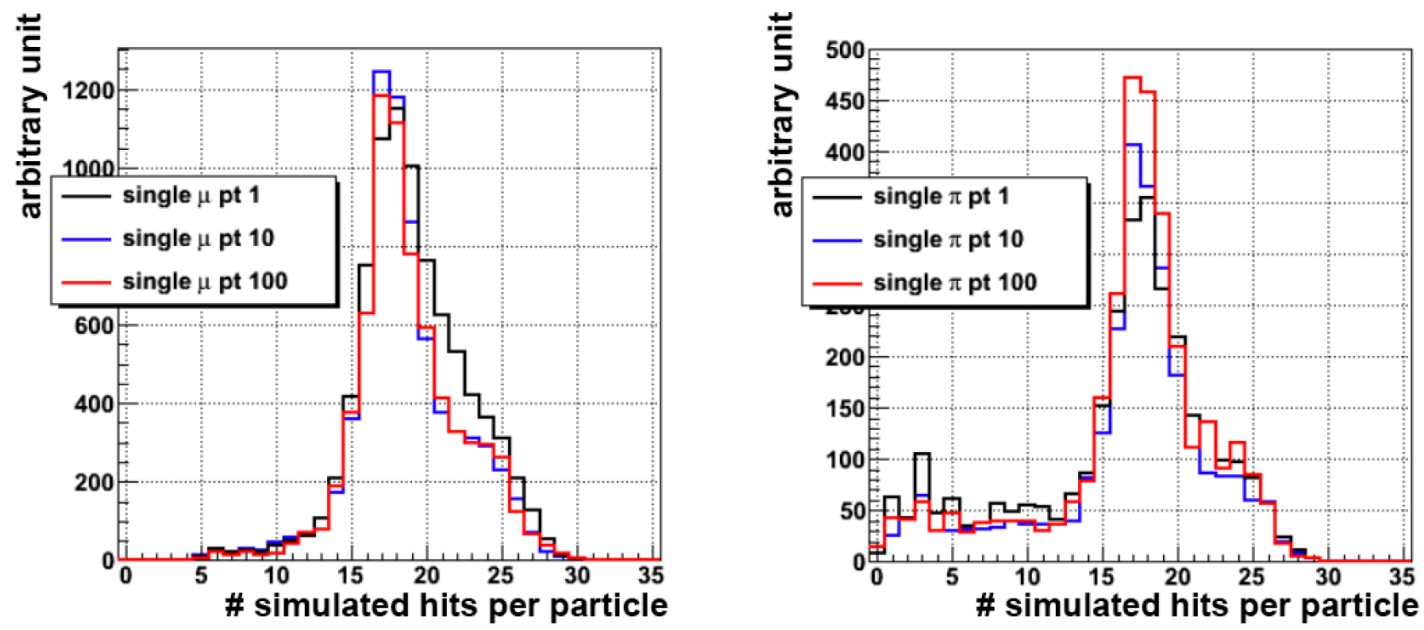

Figure 3: Number of Tracker sensors that are crossed by a simulated charged particle (SimHits). On the left are shown the distributions for 3 samples of muons with transverse momentum of 1,10 and $100 \mathrm{GeV} / c$ respectively; on the right the same three distributions for 3 analogous samples of simulated pions. Each crossed mono strip sensor provides one entry to the histograms, unlike the stereo sensors that are counted twice.

The third stage consists of a least-squares fit in the form of a Kalman filter and smoother for the final estimation of the track parameters. A "forward" fit proceeding outwards from the interaction region removes the approximations used in the track finding stage and provides an optimal estimate of the track parameters at the outside of the tracker. A "backward" fit in the opposite direction (the smoother) yields the estimate of the track parameters in the interaction region and -in combination 
with the forward fit - at each of the intermediate layers.

During the final fourth stage a quality selection is applied to the set of reconstructed trajectories in order to reject candidates that are likely to be ghost tracks: i.e. measurements due to noise, or uncorrelated particles, that are accidentally aligned along a short helical trajectory and therefore fake a genuine track. In addition to requirements on the number of hits, the $\chi^{2}$ of the fit and the energy, tracks are selected also according to their compatibility with the reconstructed vertices. While basically no vertex compatibility is requested for long trajectories with many hits and a good $\chi^{2}$, the selection cuts are very stringent for short tracks with bad $\chi^{2}$ and low $p_{t}$.
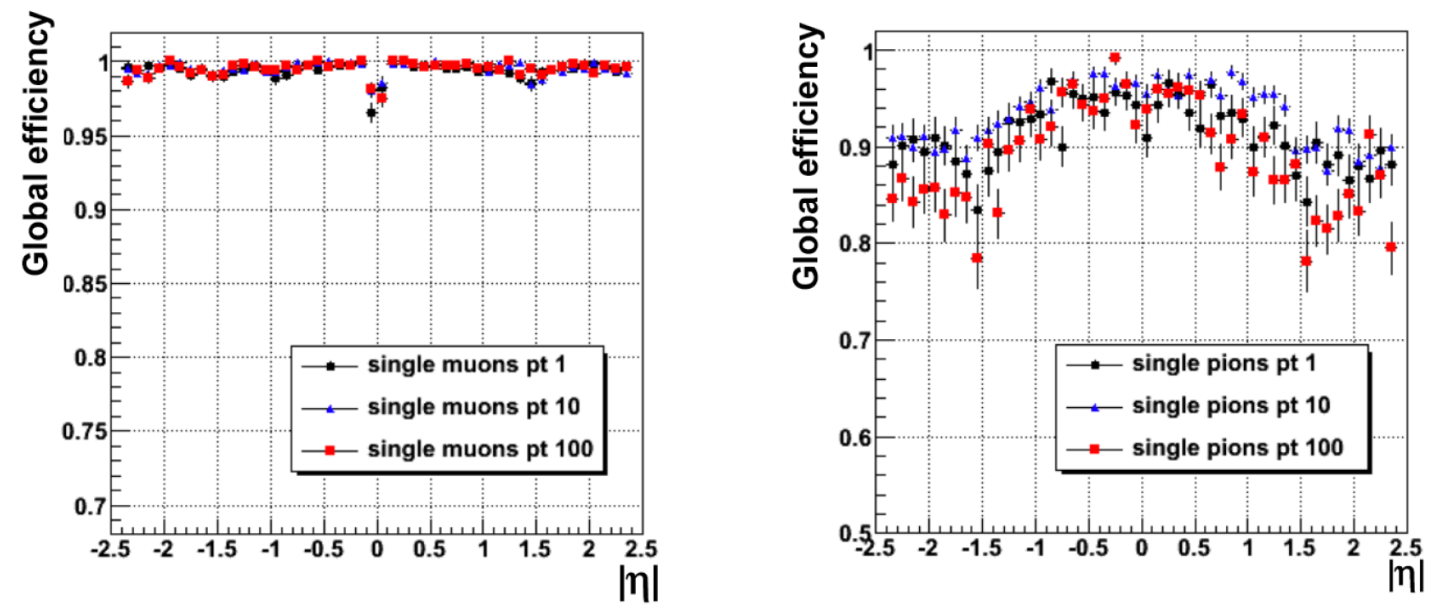

Figure 4: Tracking efficiency versus $\mathrm{h}$ for three samples $\left(p_{t}=1,10,100 \mathrm{GeV} / c\right)$ of simulated muons (left) and corresponding efficiency for three samples of simulated pions (right)

According to simulation studies, the efficiency for reconstructing muons is close to $100 \%$ in the full $\eta$ range of the geometrical acceptance of the Tracking system . Conversely, the tracking efficiency for pions varies between $85 \%$ and $95 \%$. In order to identify and reconstruct the maximum number of charged hadrons, very short tracks with as few as three hits are considered by the reconstruction software. Nevertheless many particles interact inelastically with the Tracker material even before they cross three sensitive layers: because three is the minimum number of measurements necessary to provide a standalone measure of a trajectory curvature, these particles cannot be reconstructed. The plot of the tracking efficiency versus $\eta$ for pions is clearly correlated to the distribution of the Tracker material (Fig. 2 and Fig. 4): the higher the interaction length, the lower the efficiency.

\subsection{IterativeTracking}

In order to loosen as much as possible the selection criterion while maintaining a reasonable fake rate, the sequence of four track reconstruction stages is run iteratively more than once. During a first iteration the tracks that are easier to identify are reconstructed without ambiguity: these are long trajectories with many associated hits and high momentum that are discerned from fakes without imposing any stringent vertex compatibility. All the Tracker measurements that are fully compatible with, and only with, the tracks reconstructed in the first iteration are locked and they 
are not used to identify additional trajectories. The remaining unlocked measurements are used in a second tracking iteration to reconstruct shorter and lower $p_{t}$ trajectories: the shorter the tracks, the tighter the selection. Eventually additional hits are locked and a third tracking iteration is run.

Unlike the original track reconstruction which was run in one single step, the recently implemented iterative approach allows to recover 5\% additional tracks in simulated $t \bar{t}$ events (Fig. 5). The $p_{t}$ spectrum of charged particles can be already reconstructed down to $0.5 \mathrm{GeV} / c$ (Fig. 6) and such limit is planned to be further lowered in the next version of the the tracking software.

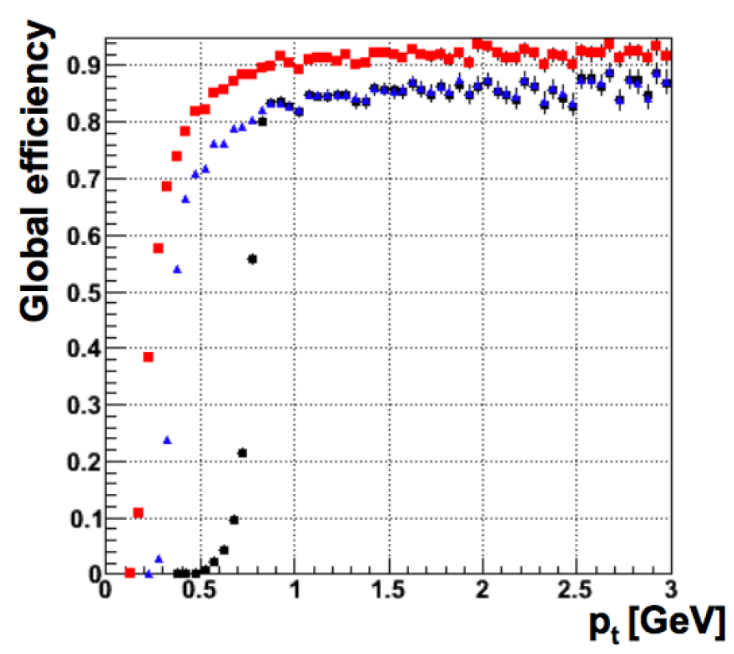

Figure 5: Tracking efficiency versus $\eta$ in the low $p_{t}$ range $(1-3 \mathrm{GeV} / c)$ for simulated $t \bar{t}$ events. The black and blue distributions refer to the original onestep track reconstruction with minimum $p_{t}$ thresholds of 0.9 and $0.3 \mathrm{GeV} / c$ respectively; the red distribution refers to the new implementation of the tracking software based on three iterations.

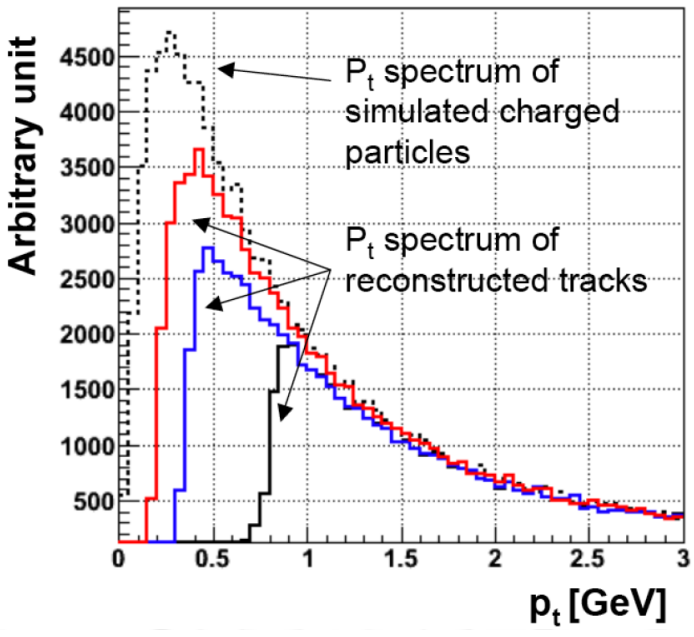

Figure 6: Transverse momentum spectrum of reconstructed charged particles for simulated $t \bar{t}$ events. The black and blue distributions refer to the original onestep track reconstruction with minimum $p_{t}$ thresholds of 0.9 and $0.3 \mathrm{GeV} / c$ respectively; the red distribution refers to the new implementation of the tracking software based on three iterations. The dashed line refers to the complete spectrum of the simulated charged particles.

After the three collections of tracks corresponding to the three tracking iterations are merged together, the resulting fake rate is lower than 2-3\% for $t \bar{t}$ events reconstructed in the barrel of the Tracker; the fraction of fakes increases to about $5 \%$ in the barrel-encap transition region where the material budget is higher and the pattern recognition is more difficult in particular for low energy particles that are affected more by multiple scattering (Fig.7).

Unlike a single step reconstruction for which as many particles as possible have to be reconstructed simultaneously, in an iterative approach each individual tracking iteration is allowed to have intrinsic inefficiencies that can be eventually compensated by the subsequent tracking iterations. Exploiting this additional handle, the three steps of the current track reconstruction sequence of CMS have been tuned to achieve the best timing performance. For simulated LHC collision events, the present 3 -steps tracking algorithm reconstructs about twice the number of tracks spending less than half the time compared to the original 1-step reconstruction. 


\subsection{Improvements to track parameters estimation}

An evident bias in the reconstructed transverse momentum of charged particles passing through the end-caps of the Tracking system has been explained as an effect of the non-uniformity of the magnetic field in these regions of the Tracker volume. Thanks to a more accurate propagator, the track fitter is now able to extrapolate the 5 trajectory parameters from one layer of the Tracker to the nearby ones taking precisely into account the variation of the magnetic field. The original bias, that was as big as $1.5 \%$ for tracks with $|\eta| \sim 1.5$, is currently smaller than $0.1 \%$ (Fig.8). To solve the equations of motion of a charged particle moving in a non-uniform magnetic field, the new track propagator uses a 4th-order Runge-Kutta numerical integration based on the adaptive method of Cash and Karp [8]. The computation of the particle trajectory is refined iteratively until the accuracy on the extrapolated particle position is better than $0.5 \mu \mathrm{m}$. The cost of the new propagator in terms of computation time is an increase of about $15-20 \%$ with respect to the overall track reconstruction timing of simulated $t \bar{t}$ events.
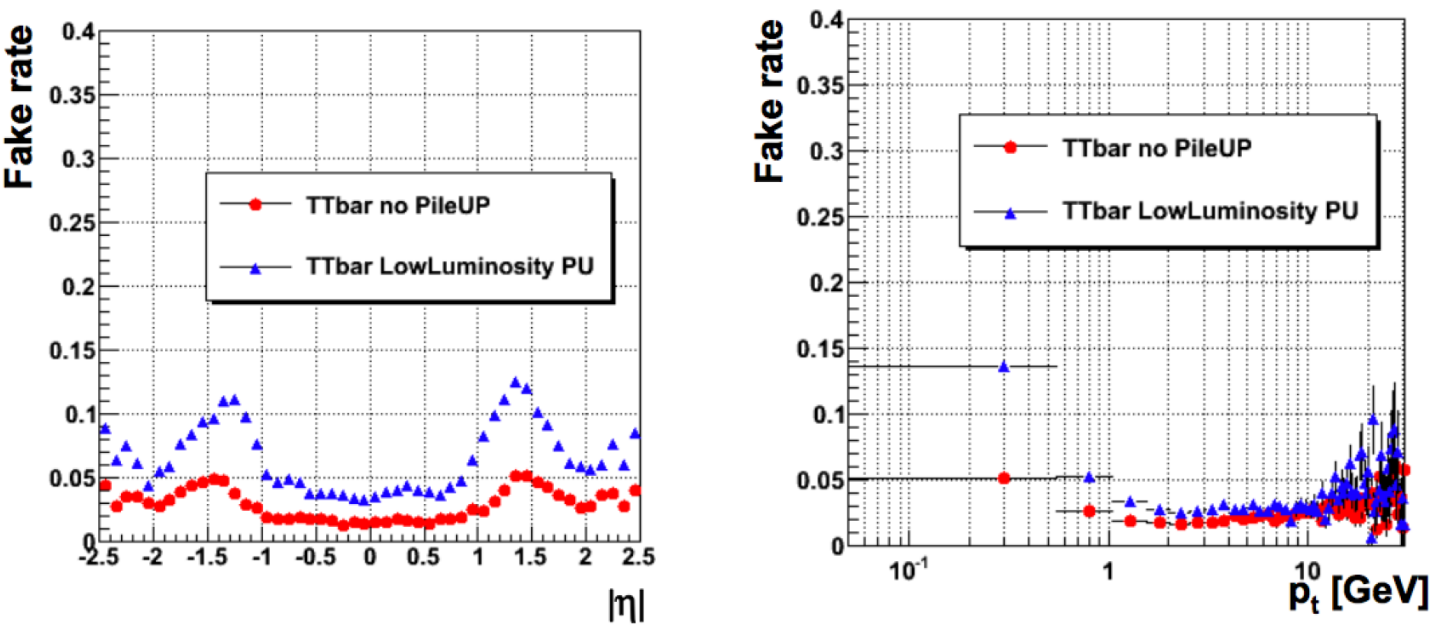

Figure 7: Rate of fake tracks versus $|\eta|$ (left) and versus the reconstructed transverse momentum (right). The red distributions refer to $t \bar{t}$ events simulated without adding the contribute of Pile Up collisions; the blue distributions refer to events simulated taking into account the contribute of Pile Up collisions for the $2 \cdot 10^{33}$ $\mathrm{cm}^{-2} \mathrm{~s}^{-1}$ LHC luminosity scenario.

Many other improvements have been recently implemented to increase the quality of the final fit of the reconstructed tracks. These enhancements include: a better estimator for the positions of pixel hits [7]; a more accurate parameterization of the material budget which is used to take into account effects of energy loss and Multiple Scattering during the track reconstruction; a tool to remove outlier measurements that are associated to a track candidate during the trajectory building even if they are actually noise hits or measurements produced by other nearby particles. Using the current version of the tracking software, the distribution of the normalized $\chi^{2}$ of fitted tracks is much closer to the expected value of 1: the average normalized $\chi^{2}$ is comprised between 0.9 and 1.1 in the full $\eta$ range, unlike the original distribution which was scattered around 1.4 (Fig.9). 


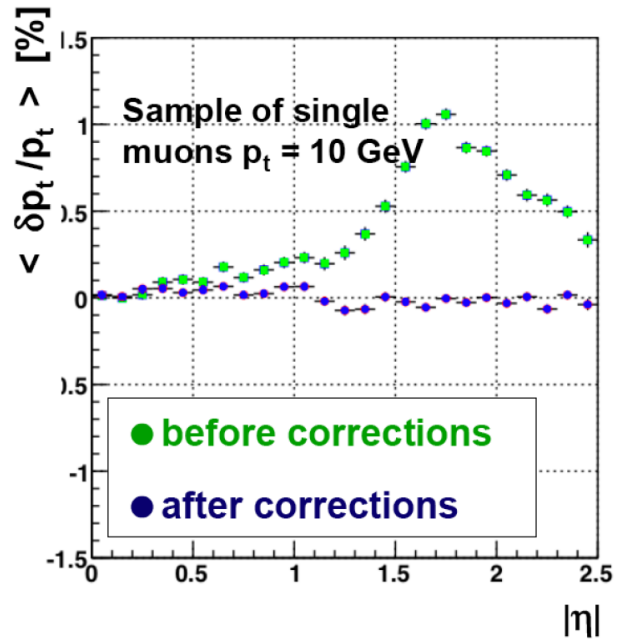

Figure 8: Bias on the estimated transverse momentum versus $|\eta|$ for simulated muons that are reconstructed without (green) and with (blue) the corrections to take into account the magnetic field non-uniformity in the end-cap regions of the Tracker.

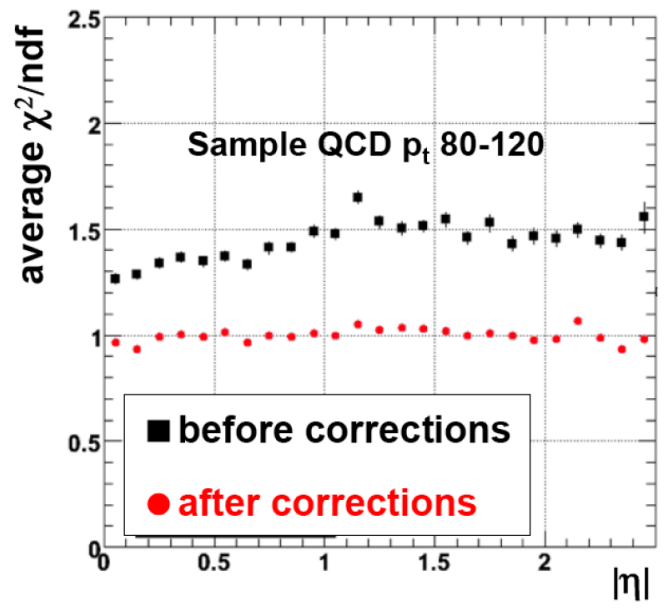

Figure 9: Average normalized $\chi^{2}$ versus $|\eta|$ for reconstructed tracks of a simulated QCD $p_{t}=80-120$ $\mathrm{GeV} / c$ sample. In black is the original distribution, in red is the one obtained using a more sophisticated fit of track parameters that, in addition to other corrections, takes better into account the nonuniformity of the magnetic field, makes use of a more accurate description of the material budget and uses a more precise estimator of the position of pixel hits.

\section{Conclusions}

The layout of the all silicon Tracker of the CMS experiment at LHC has been described, illustrating the impact of the detector's material budget on the reconstruction of charged hadrons. An overview of recent enhancements to the tracking software of CMS has been provided. The implementation of an iterative tracking procedure has been described showing the benefits for the hadron reconstruction efficiency, the reduction of fake tracks and the timing performance. It

has been shown as a more accurate description of the magnetic field non-uniformity and material distribution can improve the final quality of the reconstructed tracks.

\section{References}

[1] CMS Collaboration, The CMS experiment at the CERN LHC, JINST 3 (2008) S08004.

[2] CMS Collaboration, CMS Physics TDR Vol. 1, CERN-LHCC 001 (2006).

[3] CMS Collaboration, The Tracker System Project TDR, CERN-LHCC 006 (1998) and Addendum to the CMS Tracker TDR, CERN-LHCC 016 (2000).

[4] R. Frühwirth, Application of Kalman Filtering to track and vertex fitting, Nucl.Instr.Meth $\mathbf{A 2 6 2}$ (1987).

[5] W.Adam et al., Track reconstruction in the CMS Tracker, CMS Note 004 (2006).

[6] W.Adam et al., J. Phys. G: Nucl.Part.Phys. 031 (2005) N9-N20. 
[7] M.Swartz, D. Fehling, G. Giurgiu, P. Maksimovic and V. Chiochia, PoS Vertex2007 035 (2007).

[8] J. R.Cash and Alan H. Karp, A variable order Runge-Kutta method for initial value problems with rapidly varying right-hand sides, ACM Trans. Math. Softw. 16 (1990) 201-222. 